\title{
BMJ Open Improving substance misuse outcomes in contingency management treatment with adjunctive formal psychotherapy: a systematic review and meta-analysis
}

Luke Sheridan Rains (D) , ${ }^{1}$ Thomas Steare, ${ }^{1}$ Oliver Mason, ${ }^{2}$ Sonia Johnson (i) ${ }^{1}$

To cite: Sheridan Rains L, Steare T, Mason 0, et al. Improving substance misuse outcomes in contingency management treatment with adjunctive formal psychotherapy: a systematic review and meta-analysis. BMJ Open 2020;10:e034735. doi:10.1136/ bmjopen-2019-034735

- Prepublication history for this paper is available online. To view these files, please visit the journal online (http://dx.doi org/10.1136/bmjopen-2019034735).

Received 31 October 2019 Revised 25 June 2020 Accepted 04 August 2020

\section{Check for updates}

\section{(c) Author(s) (or their} employer(s)) 2020. Re-use permitted under CC BY-NC. No commercial re-use. See rights and permissions. Published by BMJ.

${ }^{1}$ Division of Psychiatry, University College London, London, UK

${ }^{2}$ School of Psychology, University of Surrey, Guildford, UK

Correspondence to Dr Luke Sheridan Rains; I.sheridanrains@ucl.ac.uk

\section{ABSTRACT}

Objectives Contingency management (CM) is a treatment for substance misuse that involves the provision of incentives. This review examines the hypothesis that adding another formal psychotherapy, such as cognitivebehavioural therapy (CBT) or motivational enhancement therapy (MET), to CM improves substance use outcomes at both treatment end and at post-treatment follow-up compared with CM only.

Data sources Searches were performed in December 2017 and July 2019 of seven electronic bibliographic databases (MEDLINE, PsycINF0, EMBASE, Scopus, Web of Science, CINAHL, PsycEXTRA), as well as online trial registries and EThoS, and were followed by reference list screening.

Eligibility criteria Included studies were randomised controlled trials of adults (18-65) who were using illicit substances, alcohol or tobacco. Studies featured an experimental arm delivering CM combined with a structured evidence-based psychotherapeutic intervention and a CM-only arm. Studies published up to July 2019 were included.

Data extraction and synthesis The primary outcome was biometrically verified point prevalent abstinence (PPA) at treatment end. Secondary outcomes included biometrically verified PPA at post-treatment follow-up and self-reported days of use at treatment end and posttreatment follow-up. Pooled risk ratios for PPA outcomes and standardised mean differences for days of use were calculated using random effects models. Risk of bias was assessed using the Grading of Recommendations Assessment, Development and Evaluation.

Results 12 studies $(n=1654)$ were included. The primary analysis found no evidence of a synergistic effect in PPA at treatment end (relative risk (RR) $0.97,95 \% \mathrm{Cl} 0.85$ to 1.09 $\mathrm{p}=0.57$ ). Sensitivity analysis of studies featuring CBT/MET also found no evidence of an effect (RR $0.92 ; 95 \% \mathrm{Cl} 0.79$ to $1.08 ; p=0.32$ ). None of the secondary outcomes showed any evidence of benefit.

Conclusion The results of the meta-analyses found no evidence that combining $\mathrm{CM}$ with another intervention improves the short-term or long-term effects of CM treatment.

\section{INTRODUCTION}

Contingency management (CM) is a treatment for substance misuse in which abstinence or
Strengths and limitations of this study

- This is the first systematic review to focus on the benefits of combining contingency management with another psychotherapy as a potential method for improving treatment.

- Use of the Grading of RecommendationsAssessment, Development and Evaluation approach to identify moderate overall quality of evidence across 10 randomised controlled trials.

- Lack of sufficient detail in reviewed trials to assess bias across all dimensions.

- Significant heterogeneity among the psychotherapy interventions for which content was often unclear.

- Inability to perform subgroup analysis by cognitivebehavioural therapy treatment design or other study differences such as substance or cohort.

adherence to treatment is reinforced using rewards (or less often punishments). In most cases, these are financial incentives such as a voucher or cash. Since its development in the 1960 s, a significant evidence base has been amassed demonstrating the effectiveness of CM across a variety of cohorts and behaviours. ${ }^{1-3}$ There is now good evidence of the effectiveness of CM for smoking cessation, ${ }^{4}$ alcohol $^{5}$ and substance misuse. ${ }^{1}$ Moreover, while there is solid and growing evidence base for a range of formal psychotherapies in substance use disorders, including cognitive-behavioural therapy (CBT), motivational interviewing/motivational enhancement therapy (MI/MET), mindfulness-based meditation, ${ }^{36-8} \mathrm{CM}$ is typically found to confer the greatest benefit. In a systematic review over a decade ago, Dutra $e t a \hat{l}^{3}$ found that CM was more effective at reducing use than CBT or relapse prevention treatments, but CM in combination with CBT was more effective than either CM or CBT alone. In the UK, the National Institute for Health and Care Excellence (NICE) ${ }^{9}$ recommends that drug services introduce CM programmes. 
Although CM has a strong evidence base, some have argued that any effects are likely to be short-lived as the motivational benefit of rewards will end when the rewards cease. $^{10}$ The evidence collected to date regarding the long-term benefits of CM has been mixed. Many studies that have found a benefit at treatment end fail to find an effect at follow-up. ${ }^{11}{ }^{12}$ However, other studies have found a benefit at 6 months or more post-treatment. ${ }^{11-15}$ At best, if $\mathrm{CM}$ does produce significant benefits following treatment cessation, it is inconsistent and appears to be present in only a minority of trials. In a systematic review of CM trials published between 2009 and 2014, Davis et $a l^{1}$ identified 69 trials, of which 28 included at least one follow-up assessment post-treatment. Of these, only eight found a lasting benefit of CM. Furthermore, while CM is often the most effective during treatment, other interventions more consistently produce long-term benefit. In a recent Cochrane review by Minozzi et $a l^{16}$ of psychosocial interventions for psychostimulant use, CM resulted in the clearest evidence of significant reduction in use by treatment end. However, at longest follow-up, CM produced no benefit compared with treatment-as-usual. CBT meanwhile was associated with significant reductions in use (risk ratio $=1.89,95 \%$ CI 1.18 to 3.02 ). As such, it appears that while CM is more effective during treatment, there is evidence that the benefit from other evidencebased psychotherapies appears to have less of a drop-off post-treatment.

CM is now often viewed as a method of enhancing motivation to abstain using extrinsic rewards. That is, 'to enhance and maintain initial motivation to abstain from (substance) use by providing a structure (weekly urine testing) and incentive (vouchers) for doing so'. ${ }^{17}$ This contrasts with many other structured psychotherapies. ${ }^{18}$ A central aim of MI and MET, for instance, is to improve patients' intrinsic motivation to change substance use by exploring and resolving patients' ambivalence around their substance use and building commitment to abstain. ${ }^{19}$ Meanwhile, CBT, which is frequently combined in MI/MET in trials of psychotherapy treatment for substance misuse, aims to assist the individual in tackling problematic cognitive processes and behaviours. It has been argued ${ }^{122021}$ that integrating treatments could result in improved treatment compared with treatment individually. As Sherman and McRae-Clark ${ }^{22}$ suggest: 'abstinence-based CM leads to longer periods of continuous abstinence during treatment and CBT works to enhance abstinence durability following treatment'. One explanation for the increased benefit of CM and MET/CBT combined is that each intervention is likely to address different factors influencing relapse. CM appears to be effective by increasing retention in treatment and reducing use during treatment compared with MET/ CBT alone. ${ }^{20}{ }^{21}$ Thereby, it may also be effective in terms of reducing cravings and other symptoms of withdrawal by treatment end. MET/CBT interventions meanwhile typically include aims such as 'to deal with the affective, cognitive and environmental cues that trigger drug use, and/or lack the skills to maintain abstinence'. ${ }^{12}$ These aims, if successful, could aid participants with maintaining abstinence post-treatment. A CM plus MET/ CBT intervention may therefore improve long-term treatment outcomes partially through the potentially complimentary benefits of CM and MET/CBT as standalone treatments, but second through combinatory effects, such as CM improving engagement with MET/CBT treatments compared with MET/CBT alone.

The aim of the present review is to evaluate whether substance use is improved by treatment end and by posttreatment follow-up in CM when combined with another formal, evidence-based psychotherapy, such as CBT, compared with CM alone.

\section{METHODS}

Design

The Preferred Reporting Items for Systematic Reviews and Meta-Analyses (PRISMA) statement was followed. ${ }^{23}$ The protocol was registered on PROSPERO (number CRD42017025625). ${ }^{24}$ A search of the Cochrane Database of Systematic Reviews, the Centre for Reviews and Dissemination's (CRD) database of reviews and PROSPERO found no published systematic reviews or protocols of planned reviews that were similar. This study was conducted by a team based in University College London comprised of academic and clinical researchers.

\section{Literature search}

Studies were identified by performing a keyword and subject search of the following electronic bibliographic databases: MEDLINE, PsycINFO and EMBASE using Ovid SP, as well as Scopus (Elsevier), Web of Science (WoS) and Cumulative Index to Nursing and Allied Health Literature (CINAHL). Databases of clinical trials were searched for completed and ongoing trials, which were Cochrane Central Register of Controlled Trials (CENTRAL), International Standard Randomised Controlled Trial Number Register (ISCTN registry), ClinicalTrials.gov (US National Institutes of Health), the NIHR UK Clinical Trials Gateway, and the WHO's International Clinical Trials Registry Platform (ICTRP). Grey literature was searched through the British Library's e-thesis online service (EThoS) and the APA's PsycEXTRA database. Reference lists for related systematic reviews and included publications were hand searched. Authors were contacted where needed to clarify details of studies or obtain data. Database searches were performed in December 2017 and July 2019. The search strategy for Medline (OVID) is presented in online supplemental appendix 1 .

Databases were searched from inception to July 2019. Keyword included terms related to CM, substance use (including individual illicit substances, tobacco and alcohol) and randomised controlled study design. The full search strategy for OVID SP is included in the online supplemental appendix 1. Search results were managed 
using the Endnote reference management software. ${ }^{25}$ Searches were performed by the research team.

\section{Study selection}

Studies were reviewed for inclusion by two reviewers independently. The first reviewer (LSR) removed duplicates and processed all titles and abstracts. A second reviewer (TS) sifted a random sample of $10 \%$ of unique references and checked all studies identified by the first reviewer. Disagreements were resolved by discussion between the two reviewers. Based on Cochrane guidelines, ${ }^{26}$ if there had been disagreement of $>5 \%$, further training would have been provided and screening restarted. Inclusion criteria included:

1. Only randomised controlled trials.

2. Studies that included adult participants (18-65 years old) only.

3. Study designs needed to feature at least one experimental arm in which participants received combined CM and a structured evidence-based psychotherapeutic intervention, and an arm in which participants receive $\mathrm{CM}$ alone. Studies featuring more than these two groups will also be included if the main comparison (CM plus psychotherapy compared with CM only) can be extracted from the data. Suitable psychotherapies are those listed in the International Standards for the Treatment of Drug Use Disorders by the United Nations Office on Drugs and Crime (UNODC). ${ }^{27}$ These are CBT, couples' therapy, psychodynamic therapy, behavioural therapies, social network therapy, and motivational interventions including MI and MET, and 12step facilitation for alcohol dependence.

4. Studies needed to target illicit substances, alcohol or tobacco misuse. Although financial rewards have been trialled for a range of behaviours and cohorts, there is some evidence that they affect motivation in different ways between target behaviours. ${ }^{28}$ Thus, for the purposes of this review, studies were limited to CM for substance use.

5. Studies needed to measure substance use either by a biometrically verified or self-reported measure of substance use at treatment end.

Any treatment or work setting was included. There were no restrictions on study publication dates. If necessary, reasonable efforts would have been made to translate publications into English. Publications reporting interim results or pilot studies reporting data later included in another article of the completed trial, the pilot phase/ interim publication were excluded.

\section{Data extraction and risk of bias assessment}

Data were extracted by two reviewers (LSR and TS) independently using an extraction table designed for the study and disagreements were resolved through discussion. The primary outcome was point prevalence abstinence (PPA) of the substance being targeted by the intervention at treatment end in the $\mathrm{CM}$ and the $\mathrm{CM}$ plus psychotherapy groups. PPA is a measure of abstinence from substance use measured using biometrics including urinalysis, saliva cotinine or other appropriate methods. Secondary outcomes include PPA at follow-up at least 3 months following treatment cessation and self-reported days of substance use at treatment end and follow-up. Other secondary outcomes were PPA at treatment end for treatment as usual and psychotherapy only trial arms. Data were only extracted for suitable treatment-as-usual conditions. Trials with active control interventions were not included in this secondary analysis.

For PPA outcomes, the number of substance negative samples and the total number of samples were extracted. Self-reported substance use was extracted as the mean number of substance using days. Where necessary, outcome data were calculated from the reported number of substance-positive urines or the proportion of positive/negative samples. Where data could not be extracted from the published report, the authors were contacted where possible.

Studies were evaluated for risk of bias in relation to sequence generation, allocation concealment, blinding of participants and personnel, blinding of outcome assessment, incomplete outcome data, selective reporting and other biases using the Cochrane Risk of Bias tool. ${ }^{29}$ Overall quality of evidence was assessed using the Grading of Recommendations Assessment, Development and Evaluation (GRADE) $)^{30}$ approach. GRADE assesses the quality of evidence based on five domains: (1) risk of bias; (2) imprecision (the risk of random error); (3) inconsistency (certainty in a body of evidence is highest when there are several studies that show consistent effects); (4) indirectness (evidence is most certain when studies directly compare the interventions of interest in the population of interest, and report outcome of greatest clinical interest); (5) publication bias.

Bias and quality of evidence assessment was performed by two reviewers (LSR and TS) independently and disagreements resolved through discussion. If the disagreement could not be resolved in this way, one of the supervisors of this thesis would make the decision. Funnel plots were generated to assess risk of publication bias.

\section{Data analyses}

Meta-analyses were conducted using Review Manager V.5.3. ${ }^{31}$ Data analyses used an inverse variance with random effects (Dersimonian and Laird) method throughout to compare the effectiveness of interventions between groups. For the primary analysis and secondary analyses with PPA outcomes, risk ratios were calculated. For selfreported days of substance use, standardised mean differences were calculated. Random effects analyses were used due to heterogeneity in the interventions between research reports. Due to heterogeneity in the psychotherapeutic interventions used in conjunction with $\mathrm{CM}$, a sensitivity analysis was performed in the primary analysis that included only studies delivering a CBT and/or MET intervention. Statistical heterogeneity was assessed using the $\mathrm{I}^{2}$ statistic and by visually inspecting forest plots. 


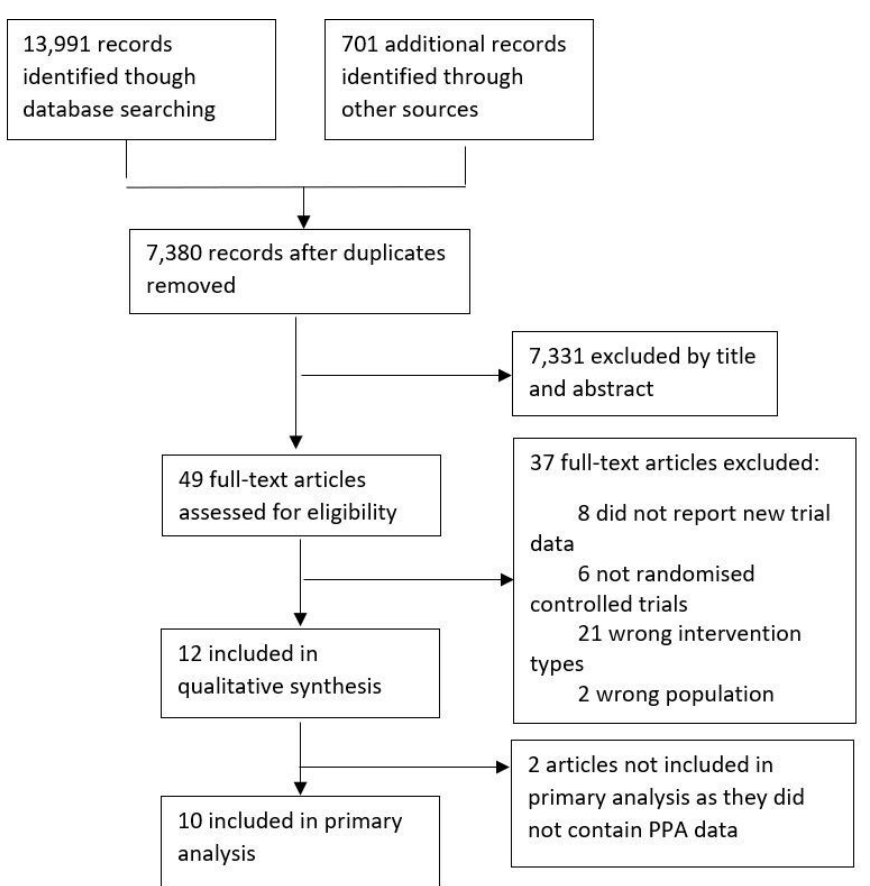

Figure 1 Study flow diagram. A total of 14692 records were initially retrieved from seven electronic bibliographic databases, online trial registries and EThoS, and reference list screening. After deduplication, 7380 unique publications were identified. Then, 7331 papers were excluded during title and abstract screening. The full texts of 49 papers were accessed. Of these, 8 did not report new trial data, 6 were the wrong study design, 21 featured the wrong intervention and 2 included the incorrect population. There were 12 studies that met the inclusion criteria, 10 of which included point prevalent abstinence (PPA) outcomes at treatment end (the primary outcome).

\section{Patient and public involvement}

There were no patients or applicable public involved in this review.

\section{RESULTS}

\section{Study selection and description}

A total of 14692 records were identified during the initial search (figure 1), yielding 12 studies that met the inclusion criteria. ${ }^{11} 2132-41$ Study characteristics are presented in table 1 . For the primary outcome, 10 studies included PPA outcomes at treatment end. Six studies included PPA outcomes at post-treatment follow-up. Six reported selfreported use data at treatment end and four at follow-up. Seven studies featured a suitable treatment as usual arm, of which five reported PPA outcomes at treatment end. Eight studies featured psychotherapy only groups and seven reported PPA outcomes at treatment end. Four studies included both psychotherapy only and TAU groups and reported PPA outcomes.

Included studies had a combined subject pool of 1654 participants. The average age was 34.28 and 1123 (68\%) were male. Eight studies had four experimental arms, two had three arms and two had two arms. Only data from suitable arms were extracted for a total subject pool of 974.

All studies were conducted in the USA, with six being recruited to and delivered via community-based substance misuse treatment centres, including four methadone clinics, one via universities, one via an agency serving homeless people, one from prenatal care clinics and three recruited using public focused advertising and delivered via a research clinic or university. Three studies targeted cocaine, two cannabis, two methamphetamine, one cocaine or methamphetamine, one cocaine or opioids, two tobacco and one polysubstance use. In five studies, all trial participants were also receiving opioid substitution therapy (four methadone and one naltrexone).

Intervention characteristics are described in table 2. The duration of the intervention period varied from 3 to 24 weeks. The number of CM sessions varied from 5 to 48 , and the maximum reward that participants could receive was between $\$ 25$ and $\$ 1277.50$. Nine studies used a variable reward schedule in which the value of the rewards rose as more sessions were passed, two studies featured a fixed reward and the other offered a prize draw for rewards of various values for each negative result.

The type of psychotherapies varied quite widely: seven included CBT and/or MET, and the other five were structured psychotherapeutic packages targeting substance use. These were:

1. Affect Regulation Treatment to Enhance Methamphetamine Intervention Success (ARTEMIS), ${ }^{32}$ an individual tailored intervention targeting positive affect.

2. Significant other or family counselling. ${ }^{33}$

3. Manualised behavioural treatment. ${ }^{35}$

4. A brief, computer-delivered intervention. ${ }^{36}$

5. One-to-one counselling. ${ }^{37}$

The duration of the psychotherapies varied from 5 to 64 sessions. The CBT and/or MET studies featured between 9 and 48 sessions of treatment over 3 to 16 weeks.

Seven studies featured follow-up assessments after the end of treatment, varying from 1 month to 1 year. For the post-treatment PPA secondary outcome, data from 6 months were included from six studies, and from 1 year from the other. Retention rates by treatment end were between $39 \%$ and $99 \%$ (median $80 \%$ ), and $65 \%$ and $95 \%$ (median $83 \%$ ) at post-treatment follow-up.

\section{Risk of bias and quality of evidence assessment}

Figure 2 summarises the risk of bias table for the included studies. None of the included trials could blind participants or personnel to allocation. Seven studies ${ }^{11213233363840}$ had low risk of bias from random sequence generation, with the risk in the other five considered unclear due to insufficient detail provided. Six studies ${ }^{11} 2132333840$ had a low risk of allocation concealment bias, while the other six had an unclear risk due to insufficient detail.

In 10 studies, it was unclear whether outcome assessors were blinded to allocation due to insufficient detail, low in one ${ }^{36}$ and high in the other ${ }^{41}$ due to the assessors not 


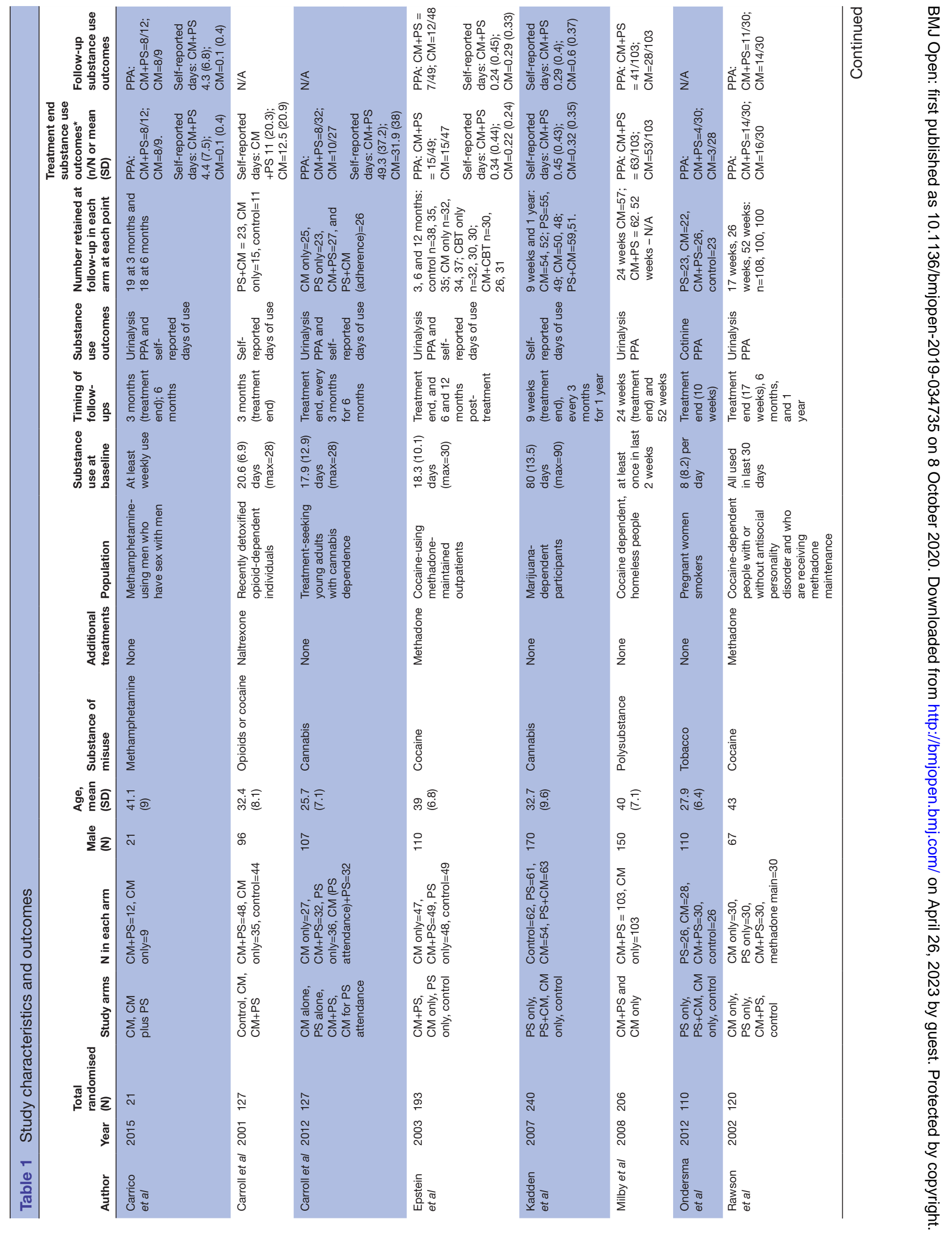




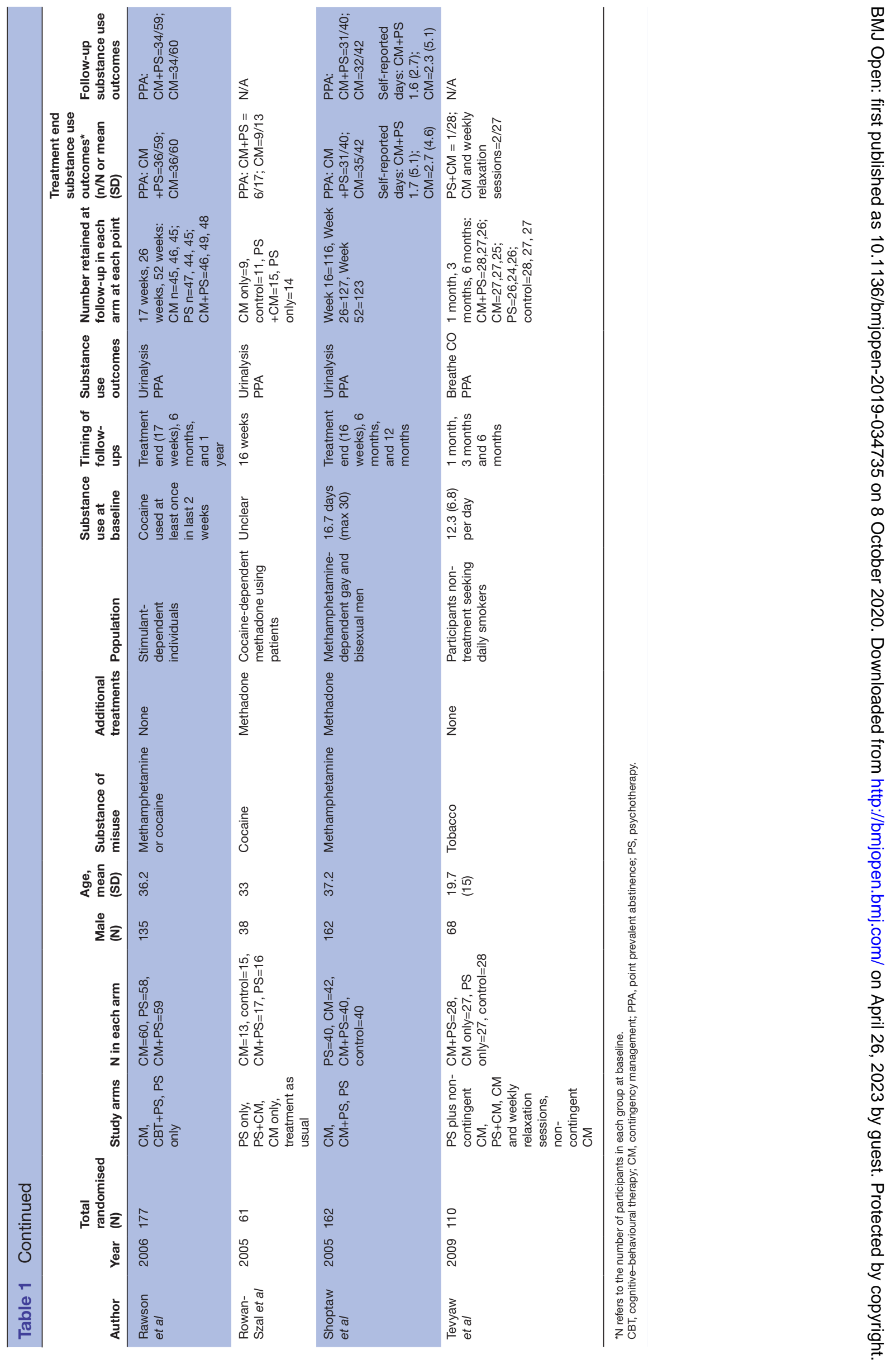




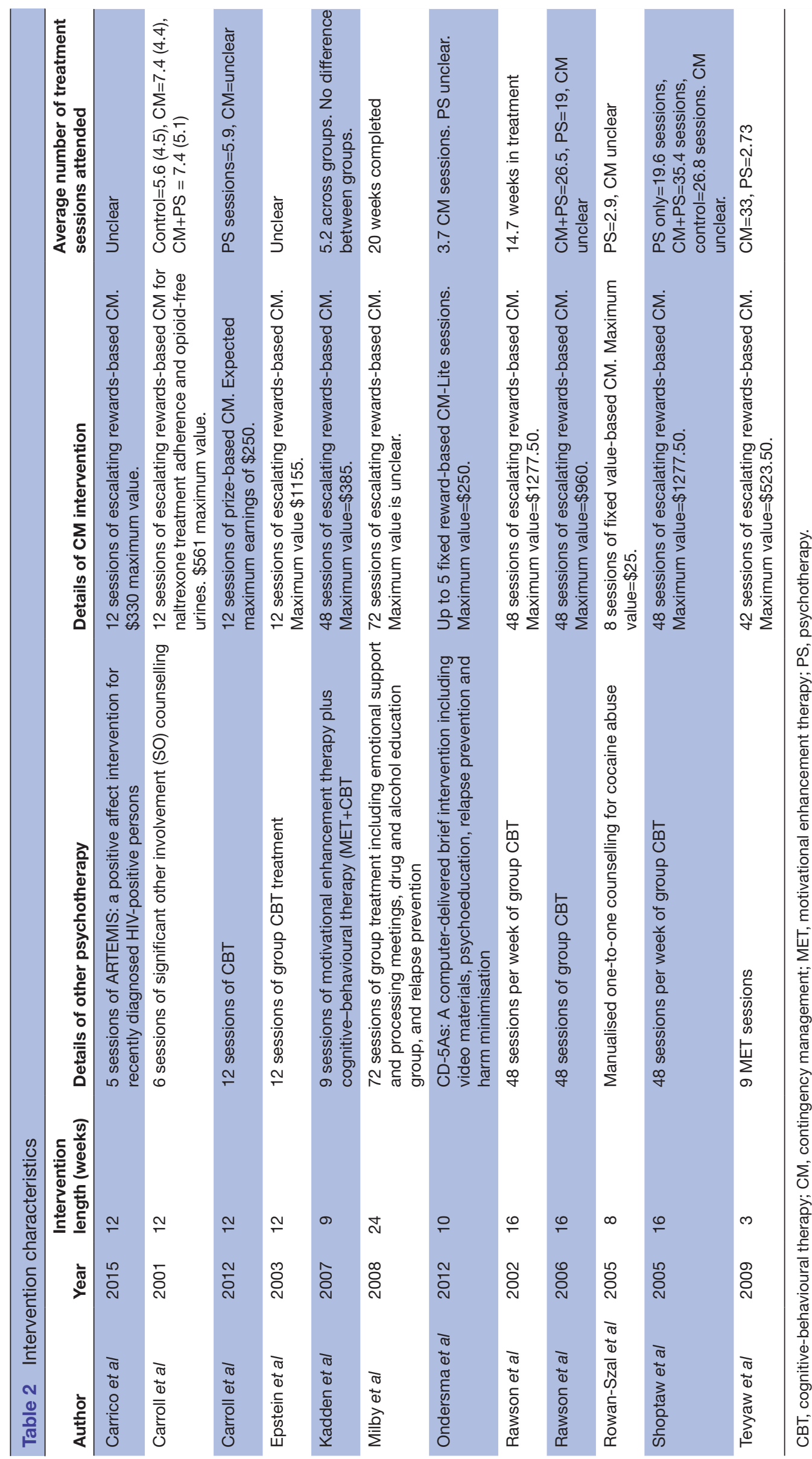




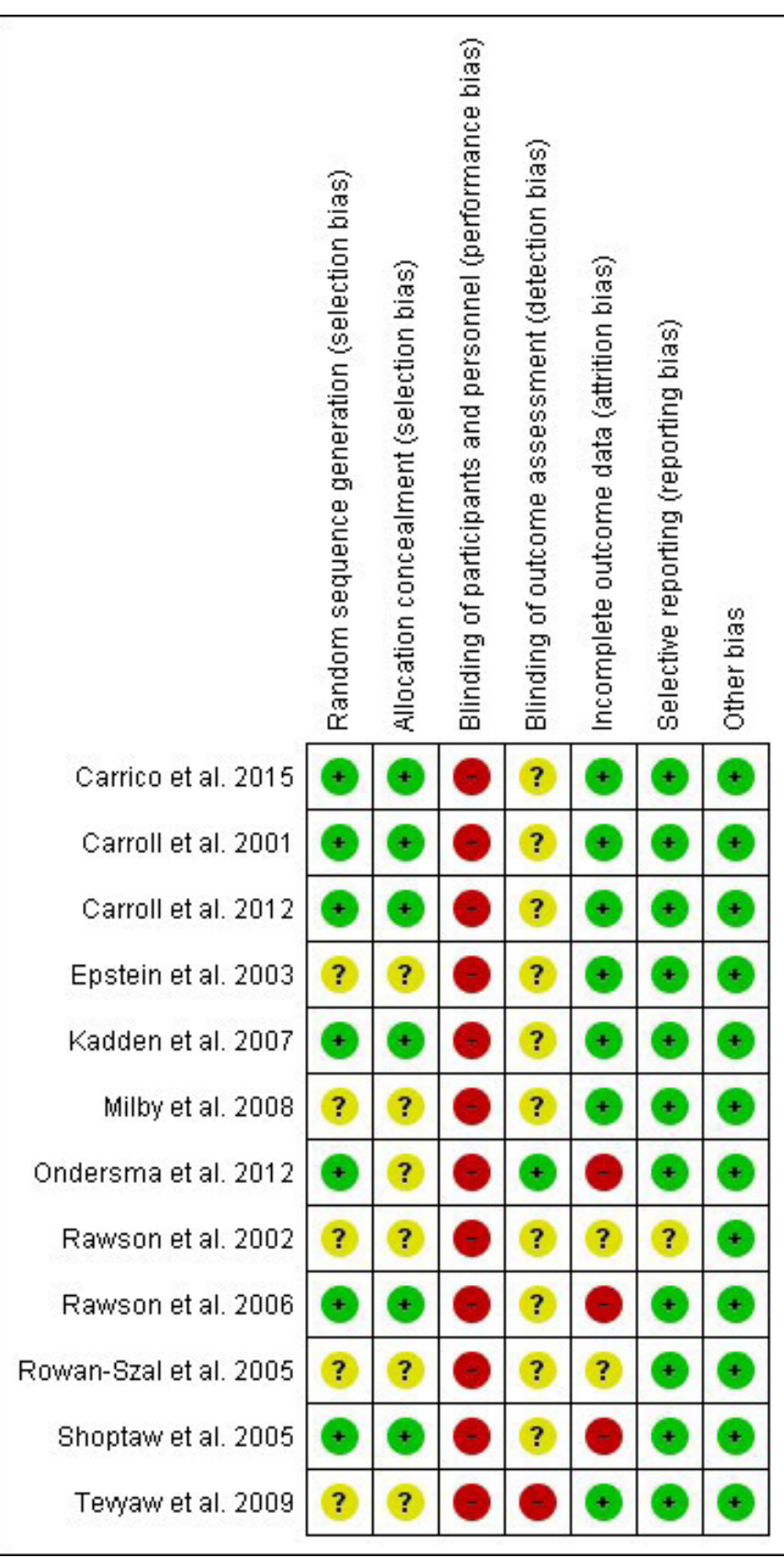

Figure 2 Risk of bias summary of included studies assessed using the Cochrane risk of bias tool.

being blinded to allocation. Three studies ${ }^{36} 38$ had a high risk of attrition bias due to poor retention rates and missingness mechanism not being explored. The risk of attrition bias in two others ${ }^{37}$ was unclear due to insufficient detail, and low in the other seven. Risk of selective reporting was low in 11 studies, and unclear in the remaining one study. ${ }^{37}$ There was a low risk of bias from the source of funding or the vested interests of researchers across all the studies. A funnel plot was generated and did not indicate publication basis (see online supplemental appendix 2).

Overall, the quality of evidence was rated as moderate using GRADE due to the possibility of bias. Risk of bias varied widely between papers, with several considered to have high risk of bias in some domains. Meanwhile, none could blind participants to allocation.

\section{Data synthesis and meta-analyses}

For the primary analysis, 10 studies reported biometrically verified (PPA) data at treatment end, including a total of 786 participants. Overall, in the primary outcome, there was no benefit on PPA substance use outcomes from adding psychotherapy to CM (relative risk (RR) $0.97,95 \%$ CI 0.85 to $1.09, \mathrm{p}=0.57$ ) (figure 3 ). There was no evidence of statistical heterogeneity $\left(\mathrm{I}^{2}=0 \%\right)$ in the included trials. The other two studies reported self-reported substance use measures only. At treatment end, neither reported an effect for CM plus psychotherapy compared with CM only. Overall, 1 out of 12 studies reported a significant treatment effect for CM plus psychotherapy compared with $\mathrm{CM}$ by treatment end, favouring CM only, and one at posttreatment follow-up that favoured CM plus psychotherapy. Due to wide variability in the types of psychotherapy used across trials, a sensitivity analysis was performed for the six trials delivering CBT and/or MET. Results were similar to the primary analysis and indicated no effect (RR $0.92,95 \%$ CI 0.79 to $1.08, \mathrm{p}=0.32$ ). As before, there was no evidence of heterogeneity in study results $\left(\mathrm{I}^{2}=0\right)$. Forest plots of all secondary outcomes are presented in online supplemental appendix 3. To address the possibility that the effectiveness of the treatment may vary between substances, a subgroup analysis was performed by substance type. Across the six studies ${ }^{32} 3437-40$ targeting stimulants, there was no evidence of an effect at treatment end (RR 0.91, 95\% CI 0.78 to $1.05, \mathrm{p}=0.19)\left(\mathrm{I}^{2}=0 \%\right)$. Other substance groups (tobacco, cannabinoids and polysubstance use) each had two studies or fewer and so were not included in the analysis.

PPA outcomes at post-treatment follow-up, like at treatment end, showed no treatment effect for CM plus psychotherapy compared with $\mathrm{CM}$ alone (RR $0.98,95 \%$ CI 0.80 to $1.21, \mathrm{p}=0.86$ ). Self-reported measures of substance use at treatment end also found no benefit for CM plus psychotherapy compared with $\mathrm{CM}$ alone $(\mathrm{SMD}=0.2,95 \%$ CI -0.0 .4 to $0.35, \mathrm{p}=0.10$ ) or post-treatment follow-up $(\mathrm{SMD}=-0.18,95 \% \mathrm{CI}-0.68$ to $0.32, \mathrm{p}=0.47)$. There was evidence of heterogeneity in the PPA results at posttreatment follow-up $\left(\mathrm{I}^{2}=34 \%\right)$, and of moderate heterogeneity in self-report at treatment end $\left(\mathrm{I}^{2}=37 \%\right)$ and high at post-treatment follow-up $\left(\mathrm{I}^{2}=77 \%\right)$.

Among studies reporting PPA outcomes, CM was more effective than treatment-as-usual by treatment end (RR $2.29,95 \%$ CI 1.45 to $3.60, \mathrm{p}<0.01) \quad\left(\mathrm{I}^{2}=0 \%\right)$. CM plus psychotherapy (RR $1.84,95 \%$ CI 1.15 to $2.95, \mathrm{p}<0.01$ ) $\left(\mathrm{I}^{2}=0 \%\right)$ and psychotherapy only (RR $1.64,95 \%$ CI 1.01 to $2.66, \mathrm{p}=0.05) \quad\left(\mathrm{I}^{2}=0 \%\right)$ were also more effective than treatment-as-usual. There was no significant difference between psychotherapy only and CM only (RR 0.80, 95\% CI 0.60 to $1.07, \mathrm{p}=0.14$ ) or $\mathrm{CM}$ plus psychotherapy (RR $0.94,95 \%$ CI 0.72 to $1.22, \mathrm{p}=0.62$ ) groups. Although there was moderate heterogeneity in results $\left(\mathrm{I}^{2}=54 \%\right.$ and $38 \%$, respectively). Five of seven studies reported CM only was 


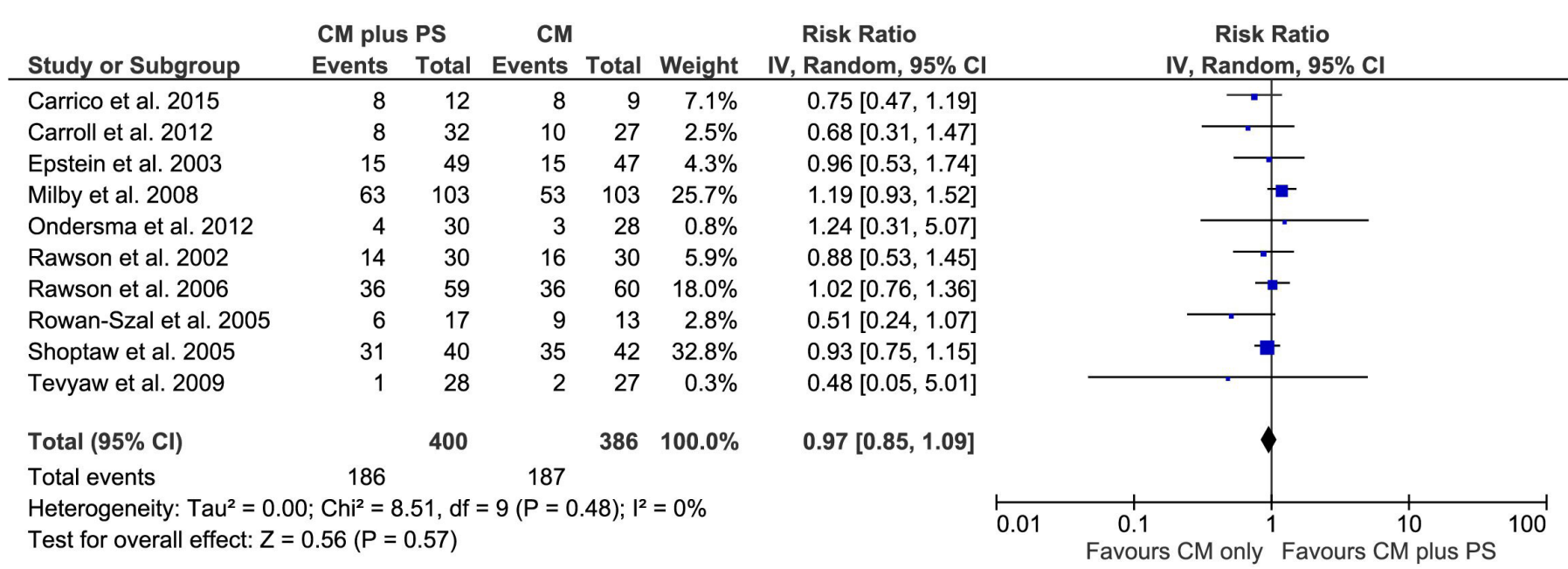

Figure 3 Forest plot of the effect of contingency management (CM) plus psychotherapy (PS) compared with CM alone on biometrically verified abstinence rates (point prevalent abstinence) at treatment end (the primary outcome). Data from a total of 786 participants were pooled. There was no evidence of benefit of adding psychotherapy to $\mathrm{cm}$ (relative risk (RR) $0.97,95 \% \mathrm{Cl}$ 0.85 to $1.09 ; p=0.57)$.

more effective than psychotherapy only. Forest plots of the results are presented in the supplementary materials.

\section{DISCUSSION \\ Main findings}

This review evaluated whether there was a synergistic effect from combining CM for substance use with a formal evidence-based psychotherapy. Twelve randomised controlled trials were identified that featured at least one group receiving CM only and another receiving CM plus psychotherapy. Overall, there was no treatment benefit for $\mathrm{CM}$ and psychotherapy compared with $\mathrm{CM}$ alone at treatment end (primary outcome) (RR $0.97,95 \%$ CI 0.85 to $1.09 ; \mathrm{p}=0.57$ ) or at post-treatment follow-up (RR 0.98 $95 \%$ CI 0.80 to $1.21, \mathrm{p}=0.86$ ). Types of adjunct psychotherapy included CBT with or without MET, MET alone, counselling, family therapy, manualised or computerised behavioural treatment and intervention aimed at improving affect. Due to the heterogeneity in treatment types, a sensitivity analysis was performed that included only the six trials with CBT and/or MET. PPA outcomes were similar and indicated no effect (RR 0.93; 95\% CI 0.79 to $1.08 ; \mathrm{p}=0.33$ ) at treatment end. These results demonstrate that it does not appear to be a benefit, as has been suggested in the literature, ${ }^{12} 21$ that there may be an additive benefit of combining CM with another formal psychotherapy, such as CBT.

Other secondary analyses found that CM provided a substantial benefit compared with treatment-as-usual and was associated with a 56\% increase in likelihood of participants being abstinent by treatment end. Similarly, both CM plus psychotherapy and psychotherapy-only groups were both more effective than treatment-as-usual, but active treatment conditions did not significantly differ when compared with each other. Overall, these patterns of results are consistent with previous literature, which strongly supports the view that behavioural treatments in general are more effective than treatment-as-usual. However, the primary result of this review indicates that there is no synergistic benefit to combining $\mathrm{CM}$ with another psychotherapy by treatment end. However, since $\mathrm{CM}$ plus psychotherapy was no better than CM alone, there appears to be a ceiling effect in the effectiveness of these treatments, such that treatment outcomes for CM cannot be improved by adding in another treatment. This pattern of results perhaps implies that both types of treatment have a similar mechanism of action. It may be that, as suggested by Davis $e t ~ a l,{ }^{42}$ the nature of the treatment is less important than receiving a formal, well-designed treatment programme.

This is the first systematic review to focus on the treatment benefit of combining CM with another psychotherapy. However, at least one other review has assessed this question as one of its outcomes. Dutra et a $\hat{l}$ analysed effectiveness by treatment end, and unlike this study, they found a benefit compared with CM only. However, they identified only two CM plus CBT trials and so caution against drawing conclusions based on this result. The present study presents stronger evidence, drawn from a wider range of interventions and a larger number of trials, and found no clear benefit. To the best of the authors' knowledge, no systematic reviews have examined post-treatment effectiveness.

\section{Limitations}

Many studies did not provide enough detail to assess bias across all dimensions. Second, no study could blind clinicians to group allocation and there are other methodological concerns with some of the trials included. Overall, the quality of evidence was moderate. The quality of evidence could be improved by more large, high-quality and wellreported trials.

Second, only a relatively small number of trials were identified, and often the trials had multiple trial arms, so the number of participants included in this review 
is relatively low. As such, some caution should be taken when drawing conclusions from these results. However, to date, the evidence indicates no overall combinatory benefit from the interventions under test. Furthermore, the secondary analyses include multiple comparisons with only a minority evidencing statistically significant differences between treatment groups. Therefore, again, these results need to be taken with a degree of caution.

There was quite high heterogeneity across the non-CM psychotherapy interventions. Although several studies included CBT or MET interventions, the number of sessions varied from 12 to 48 sessions and the contents of the different CBT interventions are often unclear. However, the sensitivity analysis of CBT or MET-only trials was performed to address this issue and also found no significant effect. However, arguably, how differences in CBT treatment designs affect impact on substance use should be explored as well. Unfortunately, there are too few studies to do this adequately. There were also too few studies to control for the potential effects of number of treatment sessions and total reward value of the CM treatment across trials. ${ }^{26}$ Doing so would help clarify the treatment benefits of the interventions.

Furthermore, a range of substances and cohorts were included, and the benefits of $\mathrm{CM}$ or psychotherapy may vary across these. Ideally, meta-analyses would explore the potentially confounding effect of differing levels of use and types of substance or differences between cohorts. However, currently, not enough data have been published. With more trials, the relationship could be explored more.

Finally, for the meta-analyses, only confirmed PPA data were used. However, all studies experienced attrition and it may be that some participants who were not followed up were in fact abstinent. While attrition does not appear to have differed substantially between the groups of interest in individual studies, it is possible that participant dropout may have biassed the results of this review.

\section{Conclusion}

The results of this paper are important for considering how to improve the substance use outcomes of $\mathrm{CM}$ treatment. Based on current evidence, adding another psychotherapy to $\mathrm{CM}$ does not improve abstinence rates at either treatment end or follow-up. However, due to the heterogeneity of the evidence base, further high-quality research is required before definitive conclusions can be made regarding the potential benefits of combining $\mathrm{CM}$ and another psychotherapy for specific cohorts.

Twitter Luke Sheridan Rains @sheridan_rains and Sonia Johnson @ soniajohnson

Contributors LSR was the principal investigator and conducted this research as part of their PhD thesis. They were involved in every stage of the research and drafted the manuscript. TS was the second rater for screening studies for inclusion, data extraction, and quality and risk of bias assessment. OM and SJ are senior academic researchers and contributed to the development of the study protocol and provided expertise throughout. All authors contributed to reviewing the manuscript drafts.
Funding The authors have not declared a specific grant for this research from any funding agency in the public, commercial or not-for-profit sectors.

Competing interests None declared.

Patient and public involvement Patients and/or the public were not involved in the design, or conduct, or reporting or dissemination plans of this research.

Patient consent for publication Not required.

Provenance and peer review Not commissioned; externally peer reviewed.

Data availability statement Data are available in a public, open access repository. Dataset available from the Mendeley repository, Dol: http://dx.doi.org/10.17632/ nypkh6shk5.1.

Open access This is an open access article distributed in accordance with the Creative Commons Attribution Non Commercial (CC BY-NC 4.0) license, which permits others to distribute, remix, adapt, build upon this work non-commercially, and license their derivative works on different terms, provided the original work is properly cited, appropriate credit is given, any changes made indicated, and the use is non-commercial. See: http://creativecommons.org/licenses/by-nc/4.0/.

\section{ORCID iDs}

Luke Sheridan Rains http://orcid.org/0000-0003-4905-4168

Sonia Johnson http://orcid.org/0000-0002-2219-1384

\section{REFERENCES}

1 Davis DR, Kurti AN, Skelly JM, et al. A review of the literature on contingency management in the treatment of substance use disorders, 2009-2014. Prev Med 2016;92:36-46.

2 Hjorthøj C, Fohlmann A, Nordentoft M. Treatment of cannabis use disorders in people with schizophrenia spectrum disorders - a systematic review. Addict Behav 2009;34:520-5.

3 Dutra L, Stathopoulou G, Basden SL, et al. A meta-analytic review of psychosocial interventions for substance use disorders. Am J Psychiatry 2008;165:179-87.

4 Giles EL, Robalino S, McColl E, et al. The effectiveness of financial incentives for health behaviour change: systematic review and metaanalysis. PLoS One 2014;9:e90347.

5 Petry NM, Alessi SM, Olmstead TA, et al. Contingency management treatment for substance use disorders: how far has it come, and where does it need to go? Psychol Addict Behav 2017;31:897-906.

6 Gates PJ, Sabioni P, Copeland J, et al. Psychosocial interventions for cannabis use disorder. Cochrane Database Syst Rev 2016;16:CD005336.

7 Cooper K, Chatters R, Kaltenthaler E, et al. Psychological and psychosocial interventions for cannabis cessation in adults: a systematic review short report. Health Technol Assess 2015;19:1-130.

8 Li W, Howard MO, Garland EL, et al. Mindfulness treatment for substance misuse: a systematic review and meta-analysis. J Subst Abuse Treat 2017;75:62-96.

9 National Institute for Health and Clinical Excellence (NICE). Clinical guideline on drug misuse: psychosocial interventions. London: NICE, 2007.

10 Petry NM. Contingency management treatments: controversies and challenges. Addiction 2010;105:1507-9.

11 Kadden RM, Litt MD, Kabela-Cormier E, et al. Abstinence rates following behavioral treatments for marijuana dependence. Addict Behav 2007;32:1220-36.

12 Litt MD, Kadden RM, Kabela-Cormier E, et al. Coping skills training and contingency management treatments for marijuana dependence: exploring mechanisms of behavior change. Addiction 2008;103:638-48.

13 Prendergast M, Podus D, Finney J, et al. Contingency management for treatment of substance use disorders: a meta-analysis. Addiction 2006;101:1546-60.

14 Carroll KM, Easton CJ, Nich C, et al. The use of contingency management and motivational/skills-building therapy to treat young adults with marijuana dependence. J Consult Clin Psychol 2006;74:955-66.

15 Stanger C, Budney AJ, Kamon JL, et al. A randomized trial of contingency management for adolescent marijuana abuse and dependence. Drug Alcohol Depend 2009;105:240-7.

16 Minozzi S, Saulle R, De Crescenzo F, et al. Psychosocial interventions for psychostimulant misuse. Cochrane Database Syst Rev 2016;9:CD011866. 
17 Budney AJ, Moore BA, Rocha HL, et al. Clinical trial of abstinencebased vouchers and cognitive-behavioral therapy for cannabis dependence. J Consult Clin Psychol 2006;74:307-16.

18 Rubak S, Sandbaek A, Lauritzen T, et al. Motivational interviewing: a systematic review and meta-analysis. Br J Gen Pract 2005;55:305-12.

19 Miller WR, Rollnick S. Motivational interviewing: preparing people for change. 2nd edn. New York: Guilford Press, 2002.

20 Litt MD, Kadden RM, Petry NM. Behavioral treatment for marijuana dependence: randomized trial of contingency management and selfefficacy enhancement. Addict Behav 2013;38:1764-75.

21 Carroll KM, Nich C, Lapaglia DM, et al. Combining cognitive behavioral therapy and contingency management to enhance their effects in treating cannabis dependence: less can be more, more or less. Addiction 2012;107:1650-9.

22 Sherman BJ, McRae-Clark AL. Treatment of cannabis use disorder: current science and future outlook. Pharmacotherapy 2016;36:511-35

23 Moher D, Liberati A, Tetzlaff J, et al. Preferred reporting items for systematic reviews and meta-analyses: the PRISMA statement. PLoS Med 2009;6:e1000097.

24 Sheridan Rains L, Johnson S, Mason O. A systematic review of the addictive effect of combining contingency management with other psychosocial interventions [online]. Available: https://www.crd.york. ac.uk/prospero/display_record.php?ID=CRD42017025625 [Accessed Mar 2019].

25 Clarivate Analytics [software]. EndNote reference software release 8.2. Philadelphia, USA, 2018.

26 Higgins J, Green S. Cochrane Handbook for systematic reviews of interventions. Cochrane book series. Wiley, 2008.

27 WHO. The united nations office on drugs and crime (UNODC) international standards for the treatment of drug use disorders [online], 2016. Available: https://www.who.int/substance abuse/ activities/msb_treatment_standards.pdf [Accessed Mar 2019].

28 Promberger M, Marteau TM. When do financial incentives reduce intrinsic motivation? comparing behaviors studied in psychological and economic literatures. Health Psychol 2013;32:950-7.

29 Higgins JPT, Altman DG, Gøtzsche PC, et al. The cochrane collaboration's tool for assessing risk of bias in randomised trials. BMJ 2011;343:d5928.
30 Bestpractice.bmj.com. What is GRADE? | BMJ best practice [online] 2019. Available: https://bestpractice.bmj.com/info/toolkit/learn-ebm/ what-is-grade/ [Accessed Mar 2019].

31 The Cochrane Collaboration. Review manager (RevMan) [Computer program]. Version 5.3. Copenhagen: The Nordic Cochrane Centre, 2014.

32 Carrico AW, Gómez W, Siever MD, et al. Pilot randomized controlled trial of an integrative intervention with methamphetamine-using men who have sex with men. Arch Sex Behav 2015;44:1861-7.

33 Carroll KM, Ball SA, Nich C, et al. Targeting behavioral therapies to enhance naltrexone treatment of opioid dependence. Arch Gen Psychiatry 2001;58:755.

34 Epstein DH, Hawkins WE, Covi L, et al. Cognitive-behavioral therapy plus contingency management for cocaine use: findings during treatment and across 12-month follow-up. Psychol Addict Behav 2003;17:73-82.

35 Milby JB, Schumacher JE, Vuchinich RE, et al. Toward cost-effective initial care for substance-abusing homeless. J Subst Abuse Treat 2008;34:180-91.

36 Ondersma SJ, Svikis DS, Lam PK, et al. A randomized trial of computer-delivered brief intervention and low-intensity contingency management for smoking during pregnancy. Nicotine Tob Res 2012;14:351-60.

37 Rawson RA, Huber A, McCann M, et al. A comparison of contingency management and cognitive-behavioral approaches during methadone maintenance treatment for cocaine dependence. Arch Gen Psychiatry 2002;59:817.

38 Rawson RA, McCann MJ, Flammino F, et al. A comparison of contingency management and cognitive-behavioral approaches for stimulant-dependent individuals. Addiction 2006;101:267-74.

39 Rowan-Szal GA, Bartholomew NG, Chatham LR, et al. A combined cognitive and behavioral intervention for cocaine-using methadone clients. J Psychoactive Drugs 2005;37:75-84.

40 Shoptaw S, Reback CJ, Peck JA, et al. Behavioral treatment approaches for methamphetamine dependence and HIV-related sexual risk behaviors among urban gay and bisexual men. Drug Alcohol Depend 2005;78:125-34.

41 Tevyaw TO, Colby SM, Tidey JW, et al. Contingency management and motivational enhancement: a randomized clinical trial for college student smokers. Nicotine Tob Res 2009;11:739-49.

42 Davis ML, Powers MB, Handelsman P, et al. Behavioral therapies for treatment-seeking cannabis users. Eval Health Prof 2015;38:94-114. 